\title{
On the variation of the crash risk with the total number of bicyclists
}

\author{
Leonhard Lücken (D)
}

\begin{abstract}
Introduction: A prominent policy, which has been proposed in many European municipalities over the last years is the promotion of cycling to decrease pollution and to increase public health. One important part of the assessment of this policy is the estimation of the induced change in bicycle crash numbers. Several recent works supported the ideas by reporting that cycling becomes safer if the number of cyclists increases, i.e., there seems to be a safety-in-numbers effect (SiN).

Methods: The problems related to the interpretation of bicycle crash and volume data are discussed and an approach aiming at a better understanding of the SiN-phenomenon is presented. In particular it is proposed to adopt models with memory to pursue causal relations and to study SiN at different time scales. To estimate daily cyclist volumes from irregular counts, a weather based model for bicycle volumes is developed.

Results: We provide a proof of concept for the proposed memory model by testing it on synthesized data and apply the proposed techniques on data provided by Berlin authorities. The application on synthetic data shows that improved fits with memory models can indicate temporal correlations within data and, thus, can give hints for causal relations. Although such a temporal correlation could not be substantiated in the real data, a surprising ambiguity was found to exist on different time scales. Over the long term, individual risks decline with increased bicycle volumes, while on shorter terms the opposite seems to be present: The more bicyclists are on the roads, the more unsafe cycling becomes.

Conclusions: The paper concludes by considering possible interpretations for the observed ambiguity. Further, a discussion of the developed methodology and some thoughts for a role that the SiN effect can play for transportation planning are included.
\end{abstract}

Keywords: Safety in numbers, Memory model, Causal relation, Bicycle crashes, Bicycle volume model, Crash risk

\section{Introduction}

Each road crash is unique in its characteristics, which include the individual series of unfortunate circumstances finally leading to a collision. Nevertheless it is important to seek patterns within the diverse set of reported road crashes to identify common factors, which help to devise measures to reduce the number and severity of crashes. In this paper we study one of the most universal functional dependencies conceivable for bicycle crash frequencies, that is, their relation to the mere volume of bicycle traffic. Several previous works have addressed this topic, (see $[6,7,10,13,14,17]$ and references therein). One of the

\footnotetext{
Correspondence: Leonhard.Luecken@dlr.de

German Aerospace Center (DLR), Institute of Transportation Systems,

Rutherfordstrasse 2, 12489 Berlin, Germany
}

main questions in this line of research has been whether the dependence of crash frequency on traffic volumes is linear or non-linear. Consider, for instance, a scenario with a constant amount of motorised traffic, where the average individual risk for a bicyclist is not correlated to the amount of other bicyclists in the scenario. In this scenario a linear dependence of crashes on the bicycle volumes would be expected.

Interestingly, it was found frequently that an increased traffic volume is accompanied by a decreased individual risk or at least a decreased risk per kilometre travelled for the traffic participants [see the aforementioned works and also Smeed [19] as one of the first studies to recognize this]. This points towards a collective retroaction of the participants on the traffic system as a whole. Indeed, if the presence of an individual bicyclist has significant 
non-local effects, which also affect situations not involving him or her directly, this would lead to a non-linear relation of crashes and bicyclist volumes. Jacobsen [13] suggested that in case of one crash participant being a vulnerable road user (VRU) the decreased risk can be a consequence of the increased visibility of a VRU travelling in a group as well as an increased attentiveness or experience of car drivers regarding the VRUs, which can be induced by more frequent encounters. More detailed distinctions of possible mechanisms have been proposed also taking into account the adaptation of novice bicyclists, who may decrease their individual risk by gaining more experience, see for instance $[11,18]$.

The hypothesis that an increased amount of interaction decreases the bicyclist's risk due to an increased awareness specifically on the side of the drivers was supported by recent results of Fyhri et al. [10,12], who reported that a decreasing risk for a collision with a car can be observed for Oslo's bicyclists during the warm season when more bicyclists are on the road while the risk for single bicycle accidents remains approximately constant throughout the whole year.

Other studies reported a negative correlation of risk and bicycle volumes on a larger time scale where the observed effect spans over various years. However, disregarding other factors than the momentary traffic volume an equally justified explanatory model could describe elevated traffic volumes as a consequence of a decreased individual risk as was pointed out by Bhatia and Wier [5]. For instance, an increased individual safety accompanied by a larger volume of bicyclists could be the expected outcome of the construction of a new cycle path along an urban road with high volumes of motorized traffic. Thus, it is important to discriminate between situations where the individual risk is reduced due to factors occurring prior to the observed change in bicyclist volumes, e.g., infrastructure improvements, and situations, where the change in bicyclist volume precedes the change in risk. It should be noted though, that the latter observation does not automatically support the above hypothesis on the driver's increased awareness as in some cases the increased safety might also be mediated by infrastructural improvements, which were implemented in response to increased bicyclist volumes. The situation where an increased safety is identified as the effect and an increased number of VRUs as the cause has been termed the Safetyin-Numbers $(\mathrm{SiN})$ effect. To the opposite causative relation we will refer as the Numbers-by-Safety (NbS) effect.

This question of causality is not treated satisfactory up to date [9] although it is of major importance for traffic planning since all derived practical implications for road safety (see Section 4) are subject to the condition that the observed effect is an SiN effect and not an NbS effect. Addressing an NbS effect in planning seems more straightforward since increasing safety by infrastructural improvements is one important objective anyway.

Since both explanatory directions, corresponding to $\mathrm{SiN}$ and $\mathrm{NbS}$, are reasonable from an a priori viewpoint it makes sense to assume that both effects can be present in a given data set. It is therefore desirable to develop a method, which allows us to differentiate between them or at least enables us to determine, which effect is stronger.

In the present work we propose a method for the identification of this causative direction. This is achieved by including memory terms into the statistical models, which, under appropriate circumstances, may indicate a temporal succession of correlated events. This is one common characteristic of cause and effect: that the cause does not lie in future of the effect. Apart from determining the dominant causative direction of the correlated phenomena, the size of the memory yielding an optimal fit can indicate the time scale of the effect. We illustrate the applicability of the method and its limits using synthetic data. A rigorous mathematical justification remains a work to be accomplished, though.

In Section 2 we develop a model, which theoretically allows to indicate non-linearities and temporally shifted correlations, and test it using synthesized data and real data on crashes and bicycle volumes in the city of Berlin in Section 3. In our analysis we disregard any other factors concerning the specific circumstances of the individual crashes than an average bicycle flow quantity.

\section{Methods}

In this section we introduce a prediction model for the number of collisions $A(t)$ between bicyclists and motorized vehicles within a time frame $t$. The only predictor variable taken into account by the model is the variation of the total volume $X$ of bicyclists in the region under consideration. This can result in an appropriate model as long as the volume of motorized vehicles stays approximately constant within the considered time span.

For the analysis we consider a dependence of the current collisions $A(t)$ not only on the current bicyclist volumes $X(t)$ but also on the average volume $X_{\tau}=\frac{1}{\tau} \sum_{i=0}^{\tau-1} X(t-i)$ in the previous $\tau$ time frames. The corresponding model is introduced in Section 2.1.

Furthermore, to apply the proposed models to empirical data on crashes and bicyclist volumes, we develop a model for the daily, weather-dependent variation of bicyclist volumes based on monthly counts at a few locations in Section 2.2. In Section 2.3, we conceive a test for the proposed method for the recovery of temporal correlations.

\subsection{Models with memory}

Several statistical works have presented results, which suggest that the assumption of a linear dependence of 
the number of collisions $A$ on the traffic volume $X$ is not appropriate. Often a non-linear relation of the form

$$
A \sim \alpha X^{\gamma}
$$

is considered instead. The reported values of the exponent $\gamma$ lie between $\gamma=0.3$ and $\gamma=0.65$ [7]. The case of linear dependence, i.e., $\gamma=1$ in (1), corresponds to a physical particle-model where entities of types $X$ and $Y$ are assumed to collide randomly with a probability $P \sim X \cdot Y$.

The SiN hypothesis sees the reason for an increased safety, i.e., a decreased risk $R=A / X$, in an increased traffic volume $X$. The main reason to postulate a causal relation of this form (and not an NbS effect, see Introduction) is that one may expect an elevated attentiveness or experience of motorists with regard to the VRUs under consideration. We introduce a measure $E(t)$ for the current magnitude of attention, resp. experience, at time $t$. The value of $E(t)$ is allowed to depend on previous $\tau$ volumes, i.e., $X(t), X(t-1), \ldots, X(t-\tau+1)$, which is indicated by adding a subscript as in $E_{\tau}(t)$, where appropriate. Further, we stipulate that in absence of any experience, i.e., $E=0$, the "particle model" is correct.

This leads us to assume a model of the form

$$
A \sim \alpha X /\left(1+E_{\tau}\right) .
$$

Below we assume that the experience $E$ is proportional to a power $\eta$ of the average volume $X_{\tau}$ within the last $\tau$ time frames. That is, we consider

$$
E_{\tau, \eta}=X_{\tau}(t)^{\eta}=\left(\frac{1}{\tau} \sum_{k=0}^{\tau-1} X(t-k)\right)^{\eta} .
$$

Note that, at least for large values of $X$, the resulting model captures (1) as a special case if $\tau=1$ and $\eta=1-\gamma$, because

$$
\frac{\alpha X}{1+E_{1,1-\gamma}}=\frac{\alpha X}{1+X^{1-\gamma}} \approx \frac{\alpha X}{X^{1-\gamma}}=\alpha X^{\gamma} .
$$

Figure 1 illustrates the most important feature of the memory model, which is an inert reaction to changes in the bicycle volumes. It shows a hypothetical time line of bicycle volumes [black curve], which exhibits an increase around $t=0$. The blue curve shows the behaviour of a model without memory $(\tau=1)$, which follows the bicycle volumes instantaneously, while the red curve illustrates the inertia of the system's adaptation to the increased volumes in the presence of a memory $\tau=10$. In both cases $\gamma=0.5$.

It seems appropriate to differentiate between different time scales for the SiN effect. Firstly, an immediate appearance of the effect would correspond to an immediately increased attention raised by the elevated presence of VRUs. For instance, an increased visibility of a group of pedestrians would have an immediate impact on their individual safety. Note that for a purely immediate mechanism a model with memory can not be expected to provide a significantly better fit.

On the other hand, if the SiN mechanism involved a longer time interval of conditioning or learning for the motorists, this would be indicated by improved model fits for the appropriate memory timespan. This equips us with a mean to deduce the time scale of the effect if it was present: It corresponds to the memory size $\tau$ that leads to the best model fit.

It seems adequate to distinguish at least between two non-immediate time scales for a possible SiN effect. The first is connected to a process of adjustment to an increased average number of VRUs due to seasonally changing conditions (on the first sunny days of spring a motorist might initially not expect many bicyclists since a longer period of low bicycle traffic volumes preceded that day). The related general behavioural adjustment, which might be hypothesized, is a change towards a more careful driving mode. This process would probably not take effect simultaneously and immediately on all drivers and therefore an inert, delayed adaptation of the traffic system as a whole would be visible in the risk if the mechanism had a significant influence on the collision frequency and no concurring factors were present neutralizing this influence.

As a process on an even slower time scale, a first familiarization with increasing numbers of VRUs due to shifts in societal mode choices would be conceivable and would express itself in the quality of models involving a larger memory.

Concerning the NbS effect, the modelling would rather aim at predicting the bicyclist volumes by past values of the individual risk, i.e.,

$$
X(t) \sim f\left(\sum_{k=0}^{\tau-1} R(t-k)\right) .
$$

For the identification of the model parameters we assume a negative binomial distribution of crashes, which allows controlling for overdispersion often observed in crash frequencies $[15,16]$.

\subsection{A bicycle volume model}

In order to study the dependence of collision frequencies on the overall bicycle traffic volumes on a level of high temporal resolution, a comprehensive record of daily bicycle volumes (usually available in the form of bicycle counts) is required.

In many municipalities, this data is rather sparse. In Berlin, for instance, counts are available only for one day per month at a few locations [see Section 3.1 for details]. In many cases the availability of weather data, which can reasonably be assumed as the most important short 


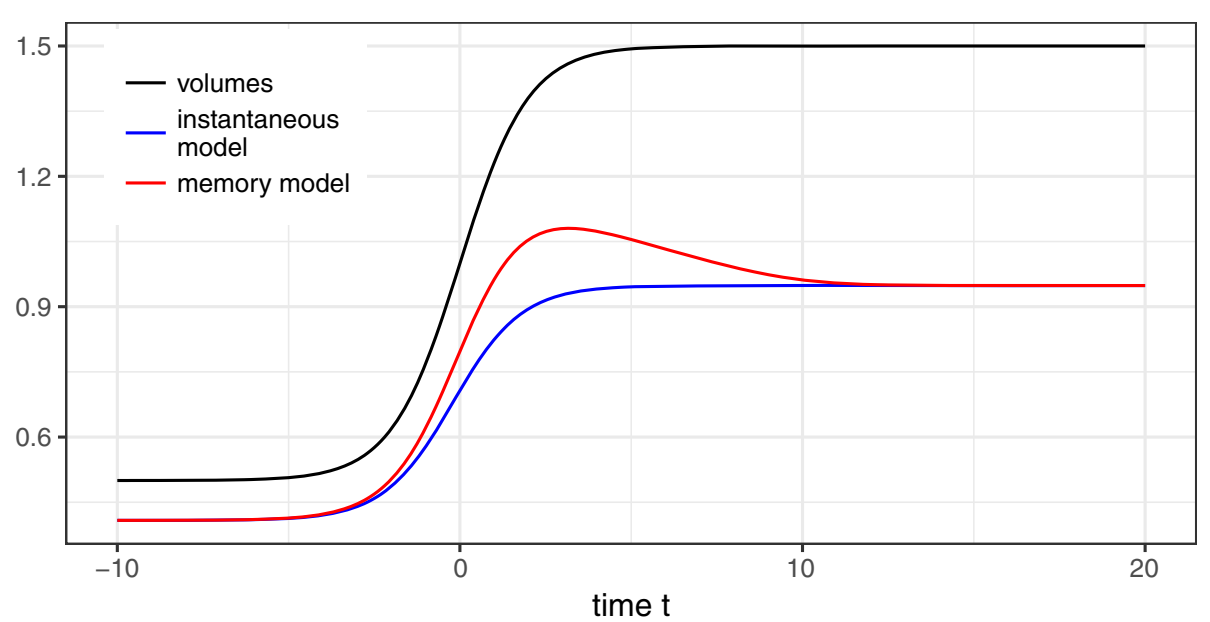

Fig. 1 Schematic illustration of the memory model (2). Including a memory $\tau>1$ has the effect of an inert response [cf. red curve] to an increase in bicyclist volumes around $t=0$ [black curve]. The model's output for $\tau=1$ is shown as a reference [blue curve]

term factor for the variation of bicycle volumes, is much more abundant, though. Here we present an approach to reconstruct daily bicycle volumes for a region of interest from sparse count data with the aid of daily weather information.

To this end we decompose the total daily bicycle volume $X(t)$ as

$$
X(t)=P(t)(1+W(t)),
$$

where $P(t)$ is a slowly varying average volume and $W(t)$ is the current deviation from the average, which is assumed to be determined predominantly by the weather.

The local values of $P(t)$ at the count locations are extracted from the available time series for the reported counts, $x_{j}, j=1,2, \ldots$, by a local regression (LOESS) $P \sim X$, see Fig. 3a. From this we obtain samples

$$
w_{j}=\frac{x_{j}}{P\left(t_{j}\right)}-1
$$

for the weather induced deviation $W(t)$, which should have a comparable magnitude across the different count locations. As predictors of $W(t)$ we considered the daily mean temperature $T(t)$ (in $\operatorname{deg} C$ ), the daily hours of sunshine $S(t)$, and the daily height of precipitation $N(t)$ (in $\mathrm{mm}$ ). In this study the functional dependence was assumed to be of the form

$$
W=\alpha+\beta f\left(c_{N} N\right) \cdot f\left(c_{T}(T-\theta)\right) \cdot\left(1+a_{S} f\left(c_{S} S\right)\right) /\left(1+a_{S}\right),
$$

where $f(x)=(1+\exp (x))^{-1}$.

\subsection{Recovering delayed correlations in synthetic data}

In this section we describe a test for the capability of the proposed memory models to reveal temporal relations in time series. For the purpose of evaluation we generated samples $z(t)$ synthetically, which were drawn from negative binomial distributions $\Gamma_{r, m(t)}$ with mean values

$$
m(t)=\frac{\alpha X(t)}{1+E_{\tau, \eta}(t)}
$$

and shape $r=10$ (parameter of the Gamma distribution in the Gamma-Poisson mixture). For the evaluation we chose $X(t)$ as the normalized (i.e., $\langle X\rangle=100$ ) daily predicted bicycle volume in Berlin with $t$ ranging over the years 2013 and 2014, and $E_{\tau, \eta}(t)$ denotes the corresponding measure of experience, see (3). The parameters $\alpha=0.55$ and $\eta=0.2$ have been fixed as we were interested in the abilities of the model to recover $\tau$. To this end, several sets of synthetic crash data were created, with $\tau$ ranging within $1, \ldots, 20$ days of memory. Qualitatively, all synthetic data sets coincided well with the observed crash numbers, see Fig. 2a-c.

As a next step, a model of the form (2) was fitted to the synthesized data with different values of $\tau=\tau_{\text {model }}$ in order to determine the capability of the modelling to recover the parameters from the data, in particular the chosen values for $\tau$. The model fit was accomplished using a maximum likelihood estimation (MLE). Hence, we sought for the maximum of

$\left(\tau_{\text {model }}, \eta, r, \alpha\right) \mapsto L\left(\tau_{\text {model }}, \eta, r, \alpha\right)=\log \left(\prod_{t} \Gamma_{r, m(t)} z(t)\right)$.

Here, the predictors $m(t)$ for the mean were given in a non-standard form as in (8) with $\tau=\tau_{\text {model }}$ and $\Gamma_{r, m(t)}(z(t))$ is the probability mass of the negative binomial distribution in the sampled value $z(t)$. 

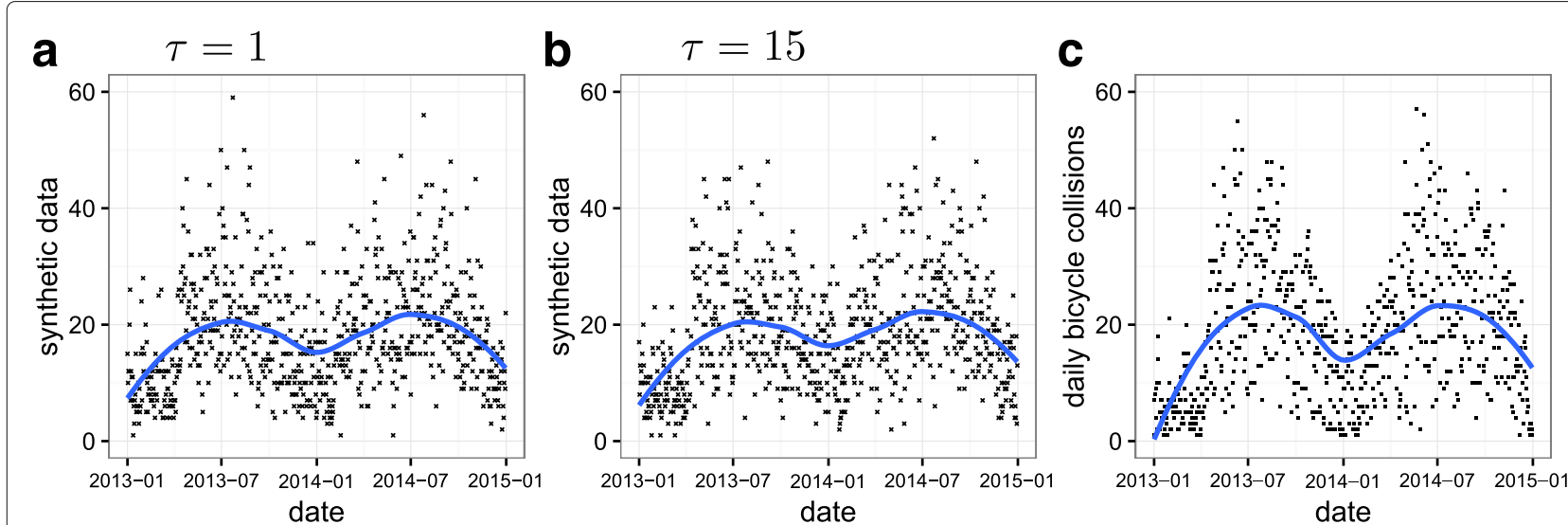

Fig. 2 Synthesized crash data [in (a) without memory and in (b) with memory] and real crash data [in (c)]. A smoothing local regression fit is shown to guide the eyes

The models usefulness for revealing temporal correlation may be assessed by its capability to recover the real value of $\tau$, which was estimated as the one leading to an optimal MLE. The results are reported in Section 3.2.

\section{Results}

In this section we first present predictions obtained for the bicycle volume model (7) in Subsection 3.1. The predictions showed an overall match with the original data on the days where bicycle counts are available.

In Subsection 3.2, we report results from the application of the memory model (2) on synthesized data, which support its possible applicability for the recovery of temporal relations.

Finally, for the real crash data from Berlin we found that different effects can be observed at different temporal resolutions in Subsection 3.3. On the annual aggregation level we observed a decrease in individual risk accompanied by an increase in bicycle volumes in Berlin during the years 2001-2014. For daily or monthly crash counts no indication of a possible SiN effect could be detected at all. Surprisingly, rather the opposite effect of increasing risk with increasing bicycle volumes seemed to prevail.

\subsection{Predicted bicycle volumes}

In this section we report on the results of an estimation of daily bicycle volumes in Berlin based on sparse count data and daily weather data.

\subsubsection{Data sources}

For Berlin, monthly bicycle counts were available from eight locations during the years 2007-2014 and less regular counts for the same locations between the years 2001 and 2006, see [2]. Apart from these bicycle counts, a general official estimate for the development of the annual average bicycle volume in Berlin [2] and data on mean temperature, hours of sunshine, and precipitation, obtained from the DWD-weather station in BerlinTempelhof [3] were used to predict the relative changes of the total bicycle volume in the whole city, see also Section 2.2.

\subsubsection{Reconstruction of daily bicycle volumes}

The parameters for the weather-based bicycle volume model (7) were determined by a least squares fit on the combined data from all count locations utilizing weather data from the Climate Data Center of the DWD [3]. We obtained: $\alpha=-0.843, \beta=2.766, c_{N}=0.063$, $a_{S}=2.576, c_{S}=-0.31, c_{T}=-0.228$, and $\theta=3.19$. The maximal $p$-value was $3.42(10)^{-4}$ for $a_{S}$. The resulting predictions (7) for the daily bicycle volumes were used to predict daily collision numbers in Section 3.2.

Figure 3 shows exemplary results for the location of Kreuzberg, where actual count data is available for comparison. The values for the normalized average bicycle volume $P(t)$ associated to the whole area of Berlin have been obtained by an interpolation of official estimates for the normalized annual volumes [2], see Fig. 5a.

\subsection{Evaluation of the memory concept}

In the following, we describe the results the recovery of delayed correlation in synthetically generated data by a memory model of the form (2), see Section 2.3. To determine the optimal MLE, we used the negative binomial model of R's MASS-library [20] to find the parameters $\alpha^{*}\left(\tau_{\text {model }}, \eta\right)$ and $r^{*}\left(\tau_{\text {model }}, \eta\right)$ for fixed values of $\tau_{\text {model }}$ and $\eta$. For the resulting function $L^{*}\left(\tau_{\text {model }}, \eta\right)=$ $L\left(\tau_{\text {model }}, \eta, r^{*}\left(\tau_{\text {model }}, \eta\right), \alpha^{*}\left(\tau_{\text {model }}, \eta\right)\right)$, maxima in $\eta$ and $\tau_{\text {model }}$ were determined numerically.

For synthesized crash data with $\tau=1, \ldots, 20$ the MLE yielded corresponding estimates $\tau_{\text {model }}$, which are shown in Fig. 4. The estimated value $\tau_{\text {model }}$ gave a good indication for the magnitude of the correlation time $\tau$ in most cases. However, it is also noticeable that the estimate 


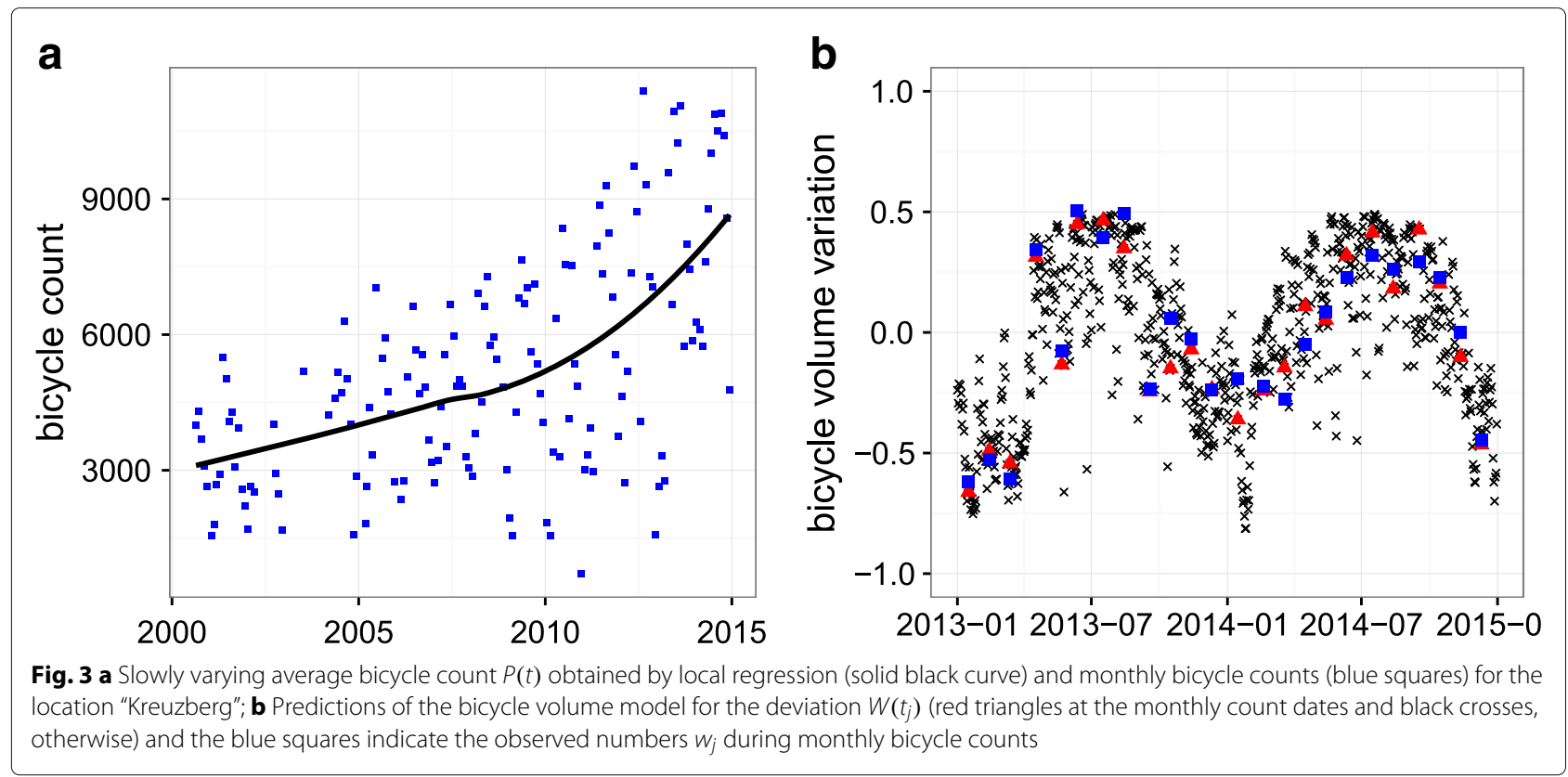

has a considerable variance with respect to the true value of $\tau$.

Thus, the value of the optimal $\tau_{\text {model }}$ seems to yield an indicator for the presence of a memory for the synthetic data although it has only limited suitability for a precise recovery of the true memory size. If a memory $\tau>1$ was used in (8) to generate the data the best estimation was also obtained for $\tau_{\text {model }}>1$. Especially for larger $\tau$ the deviation of the maximizing $\tau_{\text {model }}$ from $\tau$ is not too surprising since for larger values of $\tau$ its influence on the predictor (8) becomes weaker (the average in $E_{\tau, \eta}$ smooths its time series for longer memory times). Nevertheless, we conclude that the proposed method might bear some potential for the identification of temporal correlation in crash data if it is present to a sufficient degree.

\subsection{Application on Berlin crash data}

In this section, we report results from the application of the proposed models to crash data collected by the

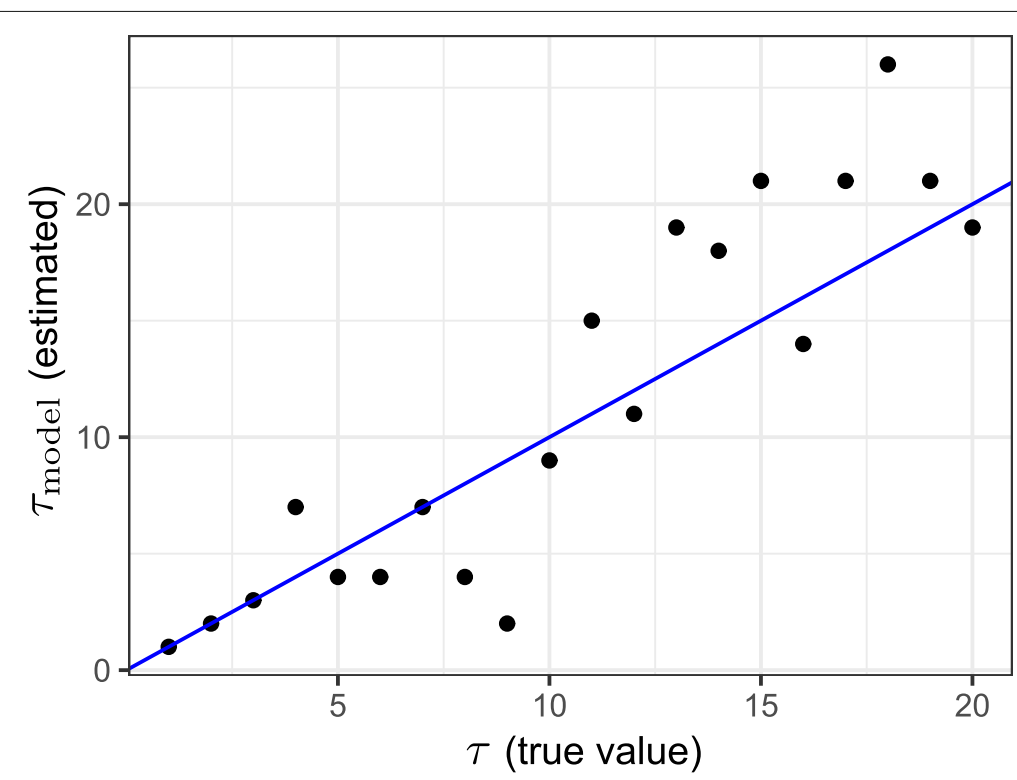

Fig. 4 Estimates $\tau_{\text {model }}$ for synthesized crash data with different true values for $\tau$. The diagonal $\tau_{\text {model }}=\tau$ has been added as a reference 
Berlin Police Department throughout the years 20012014 [1] and to data on the estimated bicycle volumes obtained from the model in Sections 2.2 and 3.1. We studied the relation between crash frequency and bicycle volumes for different levels of temporal aggregation and tested whether the models improve due to the inclusion of memory. Interestingly, different effects where found on different time scales. Over a longer period bicycle volumes increase and the individual risk decreases, while over short (seasonal) intervals the opposite effect, i.e., a positive correlation of risk and bicycle volume, is observed. For this phenomenon the term Hazard-in-Numbers (HiN) was proposed by Elvik [8]. The following table summarizes the phenomena observed on different aggregation levels:

\begin{tabular}{|c|c|c|c|}
\hline & Annual & Monthly/seasonal & Daily \\
\hline possible SiN & yes & no & no \\
\hline possible HiN & no & yes & no \\
\hline
\end{tabular}

\subsubsection{Data sources}

The the crash database provided by the Berlin Police Department [1] reports on approximately 1.37 million crashes in Berlin for the years 2001-2014 including all classes of traffic participants. Besides the spatio-temporal reference for the single crashes the data contain information on participant types, crash types, severities, circumstances, and causes. For this study we took into account 73,088 crashes between bicycles and motorized vehicles within the given period. This subset of the total crash database contains 143 fatalities, all of which are bicyclists, 5936 heavily injured (5347 bicyclists), and 54984 lightly injured (49268 bicyclists). Note that the dataset also contains information on crashes, where only material damage occurred. However, for the overall qualitative assessment in this study we disregarded any individual information on the crash type, severity, etc. We note that the volume of motorized traffic has probably varied much less than the bicycle volumes as indicated by the number of registered vehicles reported in [4]. This lead us to disregard this factor in the following.

\subsubsection{Data analysis on different time scales}

The variable quantities of risk and bicycle volumes have been normalized either to the year 2001 (in case of annual values) or to the mean over all samples (in the case of daily and monthly aggregation). For instance, a value of $X(t) \approx 130$ for the year $t=2011$ indicates an increase of the bicycle volumes by thirty percent since 2001, see Fig. 5 a.

For the different aggregation levels, the variable's values are to be considered as aggregated values over the corresponding period of time such that $X(t)$ may correspond to the normalized average bicycle volumes over the entire year, month, or day, depending on the context.

Annual aggregation On an annual level the maximal likelihood was obtained by the inclusion of a $\tau=5$ year memory. Figure $5 \mathrm{~b}$ shows the data points and the regression corresponding to $\tau=1$ and $\tau=5$. A closer look at the residuals of the predictions does not reveal a systematic improvement of predictions for larger memories [cf Fig. 5c] and an indication of temporal correlation of the hypothesized kind cannot be claimed.

However, the risks were declining within the considered period of time while the bicycle volumes were increasing, see Fig. 5a, and although no clear indication of temporal correlation was found, this leaves the possibility of a $\mathrm{SiN}$ effect on a slow time scale, i.e., that the rising bicycle volumes during the last decade have lead to a better familiarization of Berlin's drivers with the expectable behaviour of the bicyclists.

Monthly aggregation Figure 6 illustrates the results on a monthly time scale from several perspectives. In Panel (a) the normalized average monthly risks are plotted per month indicated by black crosses (14 values per month, i.e. one per month per year during the considered time span 2001-14). The mean values of the averages are indicated as blue squares and a local regression is added to guide the eye. One can observe that on average the risk seemed to be higher during the warm season. Since the estimated bicycle volumes were higher at that time as well, the relation between individual risk and bicycle volumes is reversed in comparison to the annual scale. This is also resembled by the positive slope of the risk in dependence of the volumes as shown in Fig. 6c. This phenomenon points towards the opposite of an SiN effect, i.e., a Hazard-in-Numbers effect.

Daily aggregation As for the annual and the monthly aggregation the estimate on the daily crash numbers were not improved by a non-linear fit, i.e., using a model of the form (2). Independent of the size of the memory $\tau$ the ML estimation gave $\eta \approx 0$, which did effectively set the term $E_{\tau, \eta}$ constant equal to one and signifies that the relation between volumes and crashes was linear on average. Since $E_{\tau, \eta}$ contains the memory terms the ML estimation for the negative binomial model gave the same values $\alpha \approx 0.295$ and $r \approx 4.77$ for all choices of $\tau$.

Figure 7a shows this fit and the observed daily bicycle collisions $A(t)$ in dependence of predicted volumes $X(t)$, while Panel (b) shows the same data for the risk $R(t)=$ $A(t) / X(t)$. A linear LS fit exhibited a slightly positive slope [Fig. 7b] though it was not as pronounced as on a monthly scale [Fig. 6c]. 


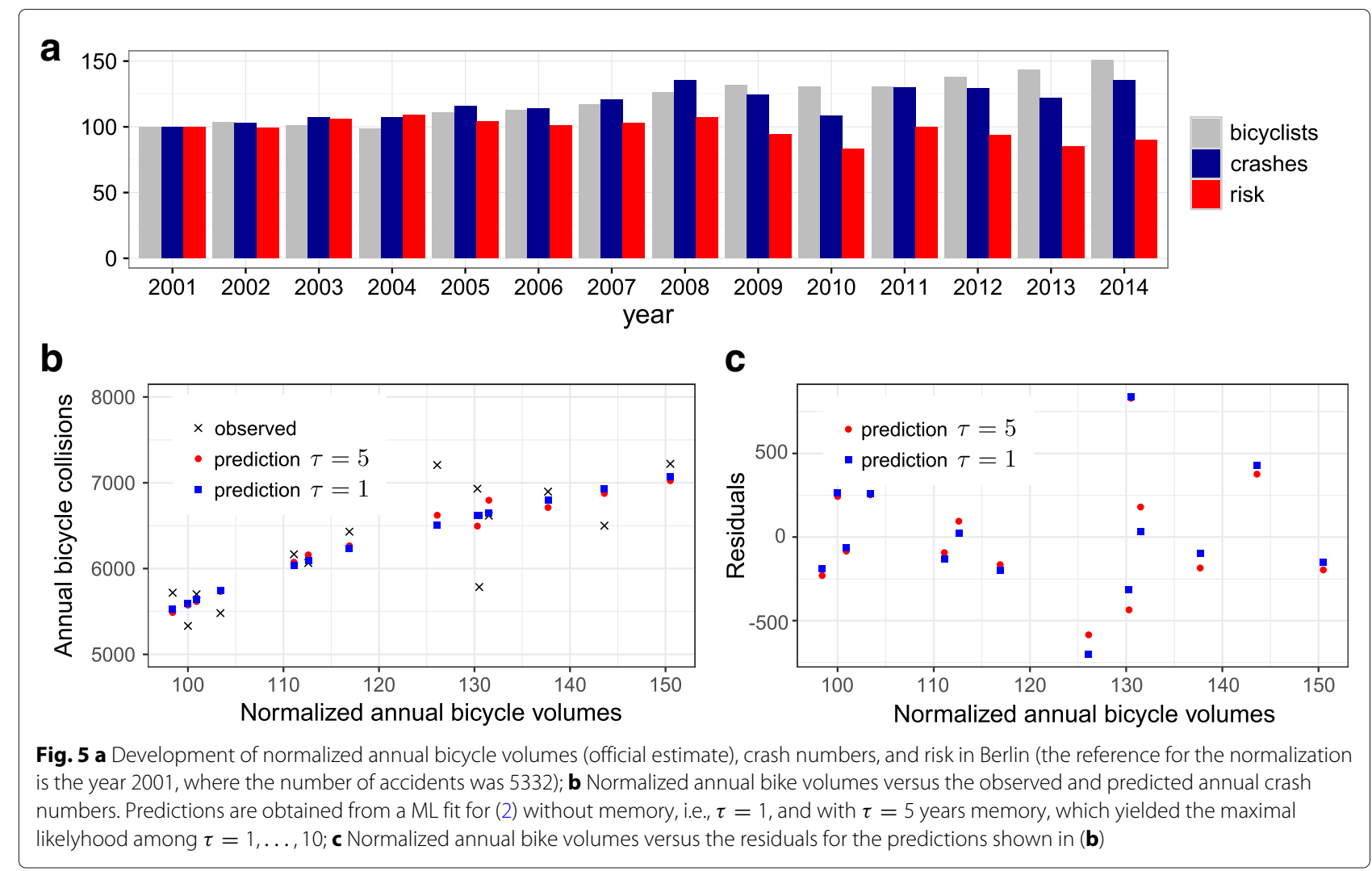

\section{Discussion}

Summary In the present work we have developed an approach to establish the existence of a SiN effect on the basis of temporally mapped data sets on bicycle volumes and crashes involving bicyclists and motorized vehicles.

Following the idea of attributing the $\mathrm{SiN}$ effect to an increased experience or attention of the motorists towards VRUs we introduced a model term to capture the evolution of this experience, see (2) and (3), and we provided a proof of concept utilizing synthesized data.

This approach generalizes previous attempts to account for the nonlinearity of the relation of volumes and collisions via the inclusion of an exponent $\gamma$ [cf. Eq. (4)]. Model (2) potentially permits to study a temporal relation between the first appearance of a change in the volume and a delayed response of the risk. Such a delayed correlation could give a significant hint to the causal relation

\section{a}

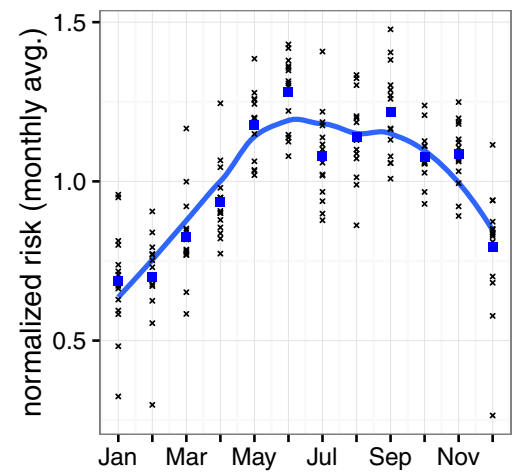

b

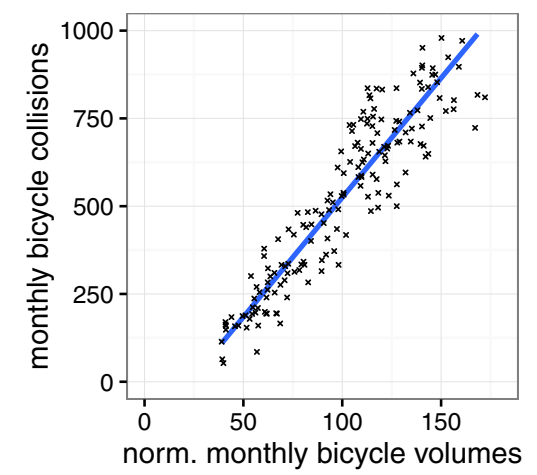

C

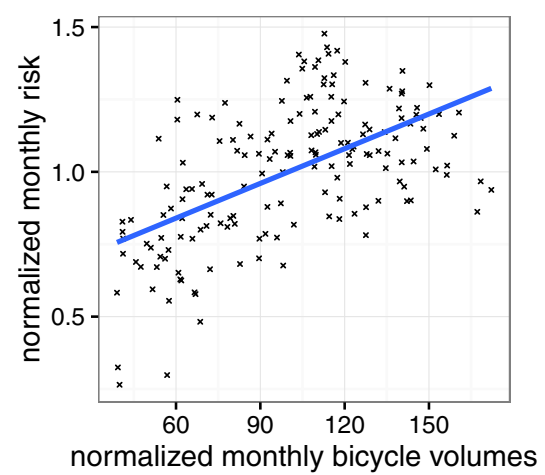

Fig. 6 a The normalized estimated risks (black crosses) per month. The total 14-year average per month is indicated by blue squares. b Linear fit (with intercept) to a negative binomial regression of the bicycle collision numbers by the normalized estimated monthly bicycle volumes. $\mathbf{c}$ Normalized estimated monthly risks with least squares fit for the same predictor as in (b) 

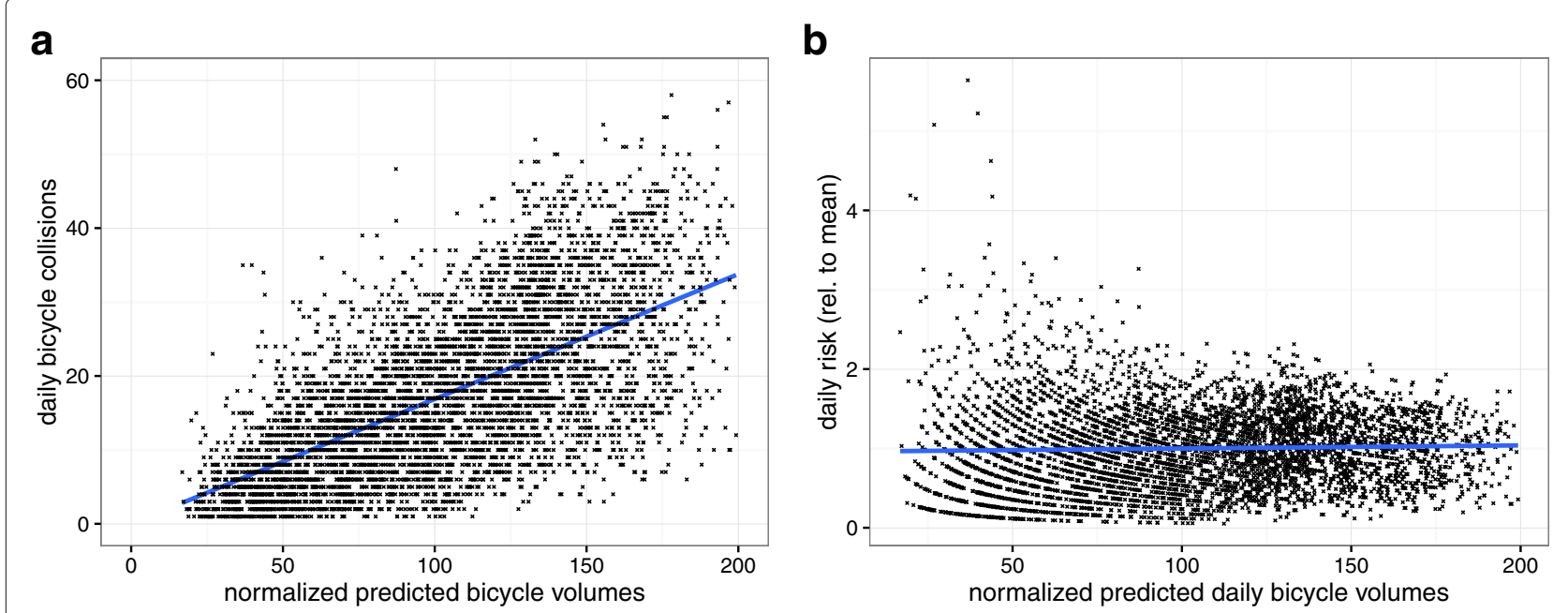

Fig. 7 Normalized, predicted daily bicycle volumes versus (a) the daily collisions involving bicyclists and motorised vehicles and (b) the corresponding daily risks

between the two quantities. The problem of determining the causality is of major importance to the identification of a true $\mathrm{SiN}$ effect because an NbS effect could likewise be responsible for a mere inverse correlation of volumes and risks [5,9]. Still a careful interpretation is needed even if the change of the risk is delayed with respect to the change of the bicycle volumes, since it does not ultimately prove the presence of an $\mathrm{SiN}$ effect. After all it might be caused by a delayed reaction of the responsible authorities in implementing a better infrastructure after increasing volumes have been recognised.

Empirical findings for Berlin We have tested the proposed memory model (2) on data sets for the city of Berlin leading to different results on different levels of temporal aggregation, see Section 3.3. For a more thorough evaluation of the model's usefulness it would certainly be helpful to apply it to different data, where a SiN-effect was suspected, since no clear indication was obtained for Berlin that delayed correlations were present between volumes and crash numbers. Despite of the rising numbers in last decades, Berlin has a long-standing tradition of bicycle traffic and for Berlin's drivers an encounter with a bicyclist is no rare event. Based on the proposed mechanism of developing correct expectations towards cyclist behaviour it may be expected that the largest $\mathrm{SiN}$ effect and thus the clearest statistical evidence could be found in areas where the amount of cyclists is very low at some point in time and increases in relation to this former state rather than in absolute numbers.

The application of the proposed model (2) on data from Berlin pointed out the interesting possibility that a $\mathrm{SiN}$ effect on a yearly scale could coexist with a HiN effect [8] on a seasonal scale. Indeed, on an annually aggregated level we found that rising bicycle volumes over the past years have been accompanied by a decreasing individual risk. However, we advise caution in the interpretation as a SiN effect since other delayed effects, as for instance infrastructural adaptations, might interfere. On the contrary, on a monthly time scale, higher bicycle volumes were accompanied by higher individual risks. A possible explanation for this finding could be an increased number of inexperienced bicyclists occurring at better weather conditions although we do not know of any studies supporting this hypothesis. We note that our results stand in contrast to results of Fyhri et al. [12] who report on a $\mathrm{SiN}$ effect in the context of seasonal fluctuations of bicycle volumes in Oslo. A higher volume of bicyclists in the warm season was found to be correlated to a lower risk of suffering a traffic collision with a motorised vehicle there.

For the careful interpretation of the results for Berlin one must also have in mind that the scarce knowledge of the volume of motorized vehicles in Berlin may exclude an important confounding factor from the model. On one hand, commuters are not regarded in the main source for the assumption of constant volumes [4]. Further, using numbers of registered vehicles as a proxy for exposure may fail if the average individual mileage did vary in the considered time span. Moreover, infrastructural measures have not been accounted for, although they might as well play and important role as a time varying factor. Thus, both factors represent possible unobserved heterogeneities, that may introduce an estimation bias [16, 21].

Memory model for crashes A possible modification for the model, which has not been verified so far, is whether a different form for the experience term (3) would result in 
an improvement of either the model itself or for the study of its properties. Possible alternatives to the term $1+X_{\tau}^{\eta}$ in (2) could be either $\left(1+X_{\tau}\right)^{\eta}$, which has the advantage of yielding the same linear model for $\gamma=0$ as for $X_{\tau}=0$, or $\exp \left(\eta X_{\tau}\right)$, giving the same value for $\eta$ independent of the scaling for $X_{\tau}$. However, both alternatives do not yield a natural definition of $E_{\eta, \tau}$ with value zero for $X_{\tau}=0$. Another possible modification, replacing the average $X_{\tau}$ by a weighted memory could result in improvements in case of an ML estimate [it does not for a non-linear LS fitting, see [17]]. It is also necessary to emphasize that the assessment of the result's significance should be done on a more rigorous level. This is no trivial task due to the nonlinearity on one hand and, on the other hand, due to the statistical dependence of the samples, which is introduced by the memory terms.

Bicycle volume model Since for Berlin there were bicycle counts available only at a few locations and at most once per month, a model for the daily and monthly variation of the overall bicycle volume in Berlin was derived on the basis of weather data. Obviously, the suitability of this model is crucial for the correctness of the derived results on the overall shape of the functional dependence between volumes and crash frequency as well as for the recovery of temporal patterns. Some additional efforts might be invested into its verification. On one hand this seems advisable due to the relatively large deviation of its predictions from the actual counts [see Fig. 3b] and also by the observation that the distribution of crashes in dependence of the bicycle volumes seems to be skewed such that a negative intercept provides a better fit [see Figs. $6 \mathrm{~b}$ and $7 \mathrm{a}$ ]. A corresponding bias towards overestimating lower bicycle volumes, which might be conceivable in Fig. 2a and b in comparison to Fig. 2c, could also induce the obtained positive correlation of volumes and risks, see Figs. $6 \mathrm{c}$ and $7 \mathrm{~b}$. Further factors that may be taken into account in a more detailed model for bicycle volumes are for example holidays, construction sites or strikes in public transport.

Practical implications There is a high demand of clear guidelines on how to include $\mathrm{SiN}$ as a factor in practical transportation planning. Especially in the context of policies that promote a shift to more sustainable transportation modes a SiN effect could serve as an argument for a shift towards cycling or walking. However, depending on the strength of the effect the increased average severity of crashes between cars and bicycles could still be contraindicative.

Further, if the immediate reason of increased visibility can be shown to be a major factor in an existing SiN effect it may be advantageous to seek the concentration of bicycle streams on major bicycle routes. In that way a situation, where several bicyclists travel as a group, might be generated more frequently and thereby provoke the supposed increase in safety arising from this situation.

A possible way to exploit an assumed increase in safety due to an increased driver's experience (even without altering the traffic volumes) could be the deliberate provocation of traffic situations, which can be considered relatively safe but require the interaction of drivers and bicyclists. For instance, the promotion of mixed traffic or shared spaces could represent adequate measures, if it can be assured that the increased number of encounters does not adversely affect safety. After all, the factor of perceived safety should not be neglected when considering such measures for increased interaction. In particular, because the alleged Numbers-by-Safety effect may in reality rather be a Numbers-by-Perceived-Safety effect.

As partly underlined by the data from Berlin presented in this work and by differing results in the literature, the $\mathrm{SiN}$ effect can not be assumed universal, i.e., it does not automatically arise if bicycle volumes are increased. The impact of an increasing number of bicyclists on the car driver's attentiveness and experience may be different from situation to situation. For instance, the learning process may saturate at some level of bicycle volume, above which further increase does not lead to significant changes in the driver's behaviour. Another important variable may be the variation of the average skill level of the bicyclists with the total volumes.

Up to date no definite method exists to predict for a given area whether a SiN effect could be expected - not to mention the prediction of its strength. Still, there is a qualitative argument for a shift towards cycling taking into account the decreasing numbers of motorized vehicles during this process: Assuming that the safety situation would improve if everybody exclusively uses the bicycle for his/her daily trips, i.e., when the shift is "complete", a "tipping point" has to exist at some value of the proportion of traffic modes beyond which the safety situation is better for the increased bicycle share. Elvik [7] has computed several values explicitly for a range of scenarios and for an assumed impact of a SiN effect.

\section{Authors' contributions}

The author read and approved the final manuscript

\section{Competing interests}

The author declares that he has no competing interest.

\section{Publisher's Note}

Springer Nature remains neutral with regard to jurisdictional claims in published maps and institutional affiliations.

Received: 28 September 2017 Accepted: 15 June 2018

Published online: 03 July 2018 


\section{References}

1. (2001) Unfalldatenbank der Polizei Berlin. Road Crash Database of the Berlin Police Department (2001-2004). Tech. rep., Polizei Berlin, PPr St II 4

2. (2014) Fahrradverkehr-Pegelzählungen Berlin - Jahresbericht 2014. Tech. rep., Verkehrslenkung Berlin (VLB). http://www.stadtentwicklung.berlin. de/verkehr/lenkung/vlb/download/bericht_radverkehr_2014.pdf, (in German). Accessed 15 Aug 2017

3. (2015) Historische tägliche Stationsbeobachtungen, Station Berlin-Tempelhof. Tech. rep., Climate Data Center of the Deutscher Wetterdienst (DWD). ftp://ftp-cdc.dwd.de/pub/CDC/observations_ germany/climate/daily/kl/historical/tageswerte_00433_19480101_ 20151231_hist.zip, (in German). Accessed 15 Aug 2017

4. (2015) Statistisches Jahrbuch 2015. Tech. rep., Amt für Statistik BerlinBrandenburg. https://www.statistik-berlin-brandenburg.de/produkte/ Jahrbuch/jb2015/JB_2015_BE.pdf, (in German). Accessed 15 Aug 2017

5. Bhatia R, Wier M (2011) Safety in Numbers re-examined: Can we make valid or practical inferences from available evidence? Accid Anal Prev 43(1):235-240

6. Ekman L (1996) On the Treatment of Flow in Traffic Safety Analysis,-a non-parametric approach applied on vulnerable road users. Bulletin/University of Lund, Lund Institute of Technology, Department of Traffic Planning and Engineering, Vol 136. Department of Traffic Planning and Engineering, Lund Institute of Technology

7. Elvik R (2009) The non-linearity of risk and the promotion of environmentally sustainable transport. Accid Anal Prev 41(4):849-855

8. Elvik R (2013) Can a safety-in-numbers effect and a hazard-in-numbers effect co-exist in the same data? Accid Anal Prev 60:57-63

9. Elvik R, Bjørnskau T (2017) Safety-in-numbers: a systematic review and meta-analysis of evidence. Saf Sci 92:274-282

10. Fyhri A, Bjørnskau T, Laureshyn A, Sundfør HB, Ingebrigtsen R (2016) Safety in Numbers - uncovering the mechanisms of interplay in urban transport. Transportøkonomisk Institutt 2016., TØI Institure, Oslo. ISBN: 9788248012566. https://www.toi.no/getfile.php?mmfileid=42927

11. Fyhri A, Bjørnskau T, Backer-Grøndahl A (2012) Bicycle helmets - A case of risk compensation? Transp Res F Traffic Psychol Behav 15(5):612-624

12. Fyhri A, Sundfør H, Bjørnskau T, Laureshyn A (2017) Safety in numbers for cyclists-conclusions from a multidisciplinary study of seasonal change in interplay and conflicts. Accid Anal Prev 105:124-133

13. Jacobsen PL (2003) Safety in numbers: more walkers and bicyclists, safer walking and bicycling. Inj Prev 9(3):205-209

14. Jonsson $T$ (2005) Predictive models for accidents on urban links-A focus on vulnerable road users. Bulletin/Lund Institute of Technology, Department of Technology and Society, Vol 226. Department of Technology and Society, Lund University

15. Liu P, Marker S (2016) Evaluation of contributory factors - effects on bicycle-car crash risk at signalized intersections, To appear in the Proceedings of the International Cycling Safety Conference 2016, Bologna

16. Lord D, Mannering F (2010) The statistical analysis of crash-frequency data: a review and assessment of methodological alternatives. Transp Res A Policy Pract 44(5):291-305

17. Lücken L, Wagner $P$ (2016) On the relation between bicycle volumes and individual risks for bicyclists in Berlin, To appear in the Proceedings of the International Cycling Safety Conference 2016, Bologna. https://elib.dlr.de/ 110827/1/Luecken_On\%20the\%20relation\%20between\%20bicycle \%20volumes\%20and\%20individual\%20risks\%20for\%20bicyclists\%20in \%20Berlin.pdf

18. Phillips RO, Bjornskau T, Hagman R, Sagberg F (2011) Reduction in car-bicycle conflict at a road-cycle path intersection: Evidence of road user adaptation? Transp Res F Traffic Psychol Behav 14(2):87-95. http:// doi.org/10.1016/j.trf.2010.11.003. http://www.sciencedirect.com/science/ article/pii/S1369847810000859

19. Smeed RJ (1949) Some statistical aspects of road safety research. J R Stat Soc Ser A (General) 112(1):1-34

20. Venables WN, Ripley BD (2013) Modern applied statistics with S-PLUS

21. Washington SP, Karlaftis MG, Mannering F (2010) Statistical and econometric methods for transportation data analysis. CRC press, New York

\section{Submit your manuscript to a SpringerOpen ${ }^{\circ}$ journal and benefit from:}

- Convenient online submission

- Rigorous peer review

- Open access: articles freely available online

- High visibility within the field

- Retaining the copyright to your article

Submit your next manuscript at $>$ springeropen.com 